\title{
Le déficit androgénique lié à l'âge chez l'homme : terminologie, épidémiologie
}

\author{
Hervé LEJEUNE \\ Département de Médecine de la Reproduction, Hôpital E. Herriot, Lyon.
}

RÉSUMÉ

Les caractéristiques de l'altération de la fonction de reproduction sont nettement différentes chez l'homme et chez la femme. Ainsi le terme d'" andropause ", volontiers employé par le grand public, est souvent remplacé par des termes plus descriptifs par la communauté médicale et scientifique. Le déclin progressif de l'imprégnation androgénique de l'organisme, qui débute dès la quarantaine, aboutit à un déficit androgénique pour une proportion des hommes par ailleurs en bonne santé. Cette proportion augmente avec l'âge. La symptomatologie peu spécifique, ne constitue qu'un syndrome d'appel nécesssitant une confirmation biologique. Le seuil permettant de définir le " déficit androgénique lié à l'âge ", correspond, dans l'état actuel des connaissances, à la limite inférieure de la normale observée chez des hommes jeunes. L'augmentation de la SHBG avec l'âge fait que le diagnostic de " déficit androgénique lié à l'âge " est plus précisément établit avec des dosages s'affranchissant des variations de la SHBG, comme le dosage de testostérone biodisponible. Le pourcentage d'hommes de plus de 55 ans présentant un " déficit androgénique lié à l'âge " passe de l'ordre de $20 \%$ avec le dosage de testostérone totale à environ $50 \%$ avec le dosage de testostérone biodisponible.

Mots clés : testostérone, vieillissement, andropause, sexualité, hypogonadisme masculin.

\section{INTRODUCTION}

On assiste à une augmentation de l'espérance de vie dans les pays développés, la conséquence en est un vieillissement de la population. Ainsi, la reconnaissance de la physiopathologie du vieillissement apparaît, plus que jamais, un enjeu fondamental. Plusieurs mécanismes peuvent être en cause dans le phénomène du vieillissement. On évoque le rôle de la réduction progressive des télomères à l'extrémité des chromosomes lors des divisions cellulaires successives. Ceci pourrait expliquer que les cellules somatiques ont un potentiel de division limité. Le vieillissement cellulaire peut, en outre, correspondre à l'accumulation progressive de mutations dans l'ADN. L'accumulation dans l'organisme de « déchets » peut aussi intervenir dans le vieillissement des organes, d'autant que ces déchets pourraient être à l'origine de mécanismes auto-immunitaires. Enfin, à l'échelon de l'organisme, on constate des altérations hormonales associées au vieillissement. À l'heure actuelle, les liens de cause à effet entre les différentes altérations constatées au cours du vieillissement ne sont pas encore complètement établis. La connaissance de l'enchaînement exact des événements aboutissant au vieillissement est pourtant importante dans la mise au point de stratégies visant à retarder les effets néfastes de l'âge.

\section{TERMINOLOGIE}

La diminution de plusieurs hormones a été mise en évidence chez les sujets avançant en âge. Ces déficits hormonaux sont volontiers dénommés en utilisant le suffixe " pause ». Ainsi, comme on parle de "ménopause » pour

Correspondance :

Hervé LEJEUNE, Laboratoire de Biologie de la Reproduction, Faculté de Médecine, Domaine Rockefeller, 8 avenue Rockefeller, 69373 Lyon, Cedex 08. 
l'arrêt de la fonction ovarienne, on utilise le terme de " somatopause " pour la diminution de la sécrétion d'hormone de croissance et d'IGF-I, d' « adrénopause » pour la diminution de production de DHEA et la baisse de son sulfate dans la circulation. Ainsi le terme d' " andropause » a pu être proposé pour décrire la diminution des fonctions testiculaires survenant avec l'âge.

Ménopause et andropause correspondraient ainsi à l'altération de la «fonction de reproduction », ce qui, au sens large, englobe la fertilité, la sexualité et les fonctions contrôlées par les stéroïdes sexuels. Toutefois, les caractéristiques de l'altération de la fonction de reproduction sont nettement différentes chez l'homme et chez la femme. Alors que la ménopause touche toutes les femmes, survient dans la grande majorité des cas entre 45 et 55 ans et correspond à un arrêt assez brutal et définitif de la fonction de reproduction avec arrêt pratiquement complet de la sécrétion ovarienne de progestérone et d'œstrogènes, il est maintenant assez clairement établi que l'« andropause » est un phénomène beaucoup plus progressif, au point qu'un déficit androgénique ne survient que chez certains sujets et le plus souvent après 60 ans. En outre, le déficit ne correspond qu'à une diminution partielle de la sécrétion de testostérone qui est moins importante que chez le sujet jeune mais n'est pas complètement nulle. Par ailleurs, on connaît chez l'homme la conservation des possibilités de reproduction jusqu'à un âge avancé.

Ces différences dans les caractéristiques du vieillissement de la fonction de reproduction entre l'homme et la femme font que le terme d' « andropause » est controversé. Certains expliquent que l' « andropause n'existe pas » puisqu'il n'existe pas d'arrêt total et définitif de la fonction de reproduction chez l'homme, comme à la ménopause chez la femme. D' autres lui reconnaissent droit de citer, à condition que les caractéristiques de l'altération de la fonction de reproduction soient reconnues comme différentes chez l'homme et chez la femme. Pour tenter de couper court à la controverse sémantique, les spécialistes vont préférer éviter le terme d' " andropause » pour utiliser une terminologie plus descriptive des caractéristiques spécifiquement masculines du vieillissement gonadique. $\grave{A}$ titre indicatif, nous avons mené une recherche sur internet, en parallèle sur un moteur de recherche généraliste (Google) et sur une base de données médicale (PubMed). Le Tableau 1 montre que les spécialistes médicaux et scientifiques donnent la préférence aux termes descriptifs, alors que les sites « grand public » préfèrent le terme « andropause ».

Les divers termes employés dans la littérature médicale en langue anglaise sont : "Andropause » ou parfois «Viropause » selon la racine grecque ou latine choisie, les termes de « Male menopause » ou « Male climacteric » sont parfois utilisés, mais alors souvent dans un but provoca-
Tableau 1 : Utilisation des termes "Andropause " et "ADAM » dans les sites internet (moteur de recherche généraliste Google) et les références bibliographiques médicales (PubMed). (ADAM = Androgen AND [Deficiency OR Decline] AND Aging AND Male).

\begin{tabular}{lcc}
\hline & $\begin{array}{l}\text { Moteur de recherche } \\
\text { généraliste(Google) } \\
\text { (nombre de sites ; \% } \\
\text { du total) }\end{array}$ & $\begin{array}{l}\text { Moteur de recherche } \\
\text { spécialisé (PubMed) } \\
\text { (nombre de références } \\
\% \text { du total) }\end{array}$ \\
\hline «Andropause» & $6230(67,3 \%)$ & $70(16,4 \%)$ \\
les 2 & $378(4,1 \%)$ & $23(5,4 \%)$ \\
«ADAM » & $3410(36,8 \%)$ & $380(89,0 \%)$ \\
\hline
\end{tabular}

teur ; les termes plus descriptifs «Androgen Decline in the Aging Male » ou « Androgen Deficiency in the Aging Male » ont pour abréviations le jeu de mot «ADAM. »; le caractère partiel du déficit androgénique peut être annoncé en utilisant le terme «PADAM » pour « Partial Androgen Deficiency in the Aging Male ».

Dans la littérature médicale en langue française, on trouve des locutions à visée descriptive utilisées pour éviter le terme « andropause », comme «déficit ou déclin androgénique de l'homme âgé ou de l'homme vieillissant ». La terminologie de « déficit androgénique lié à l'âge »souligne le fait que le déficit androgénique est dû à l'âge en luimême et non pas à une autre cause d'hypogonadisme, telle que celles que l'on peut rencontrer chez le sujet jeune.

\section{EPIDÉMIOLOGIE}

La détermination de la fréquence du «déficit androgénique lié à l'âge » nécessite de faire le rapport :

\section{nombre de sujets présentant un «déficit androgénique lié à l'âge » \\ nombre de sujets de la population étudiée.}

Le résultat sera variable selon le numérateur et selon le dénominateur, ce qui revient à définir des critères diagnostiques du « déficit androgénique lié à l'âge » pour le numérateur et préciser, pour ce qui est du dénominateur, la population dans laquelle on recherche les sujets présentant un « déficit androgénique lié à l'âge ».

\section{Critères de « déficit androgénique lié à l'âge »}

La symptomatologie clinique de l'hypogonadisme acquis de l'adulte (Tableau 2) est peu spécifique et ainsi peu utilisable comme critère dans des études épidémiologiques. C'est le dosage de la testostérone qui a permis de montrer, tout d'abord dans des études transversales, le phénomène du « déficit androgénique lié à l'âge ", en mettant en évidence une diminution statistique de la testostérone circulante avec l'âge, chez des hommes d'âge varié, mais tous en 
Tableau 2 : Symptômes du déficit androgénique chez l'homme adulte

- Diminution de la Libido

- Troubles de l'érection

- Dépression de l’humeur

- Asthénie physique el psychique

- Diminution de la masse et de la force musculaire

- Augmentation de la masse grasse

- Réduction de la pilosité à topographie masculine

- Réduction de la spermatogenèse. du volume et de la consistance testiculaire

- Gynécomastie

- Anémic

- Ostéoporose

bonne santé [4]. Cette notion de diminution de la testostérone circulante avec l'âge vient d'être précisée de manière longitudinale sur un nombre élevé de sujets suivis de manière prospective [5]. Il s'avère que le déclin de la testostérone circulante débute tôt dans la vie, dès la fin de la trentaine et se poursuit à un taux constant par la suite. Toutefois ce déclin n'aboutit à un déficit que chez certains sujets. Ainsi, une proportion des hommes âgés ont une testostéronémie inférieure à la limite inférieure des valeurs observées chez les hommes jeunes. C'est à partir de cette constatation qu'est né le concept du « déficit androgénique lié à l'âge » qui touche seulement une partie des hommes, considérés par ailleurs comme «en bonne santé ".

La définition des critères permettant de faire le diagnostic de cet état de "déficit androgénique lié à l’âge » comporte en fait deux questions, 1) le choix du type de dosage le plus adapté pour faire le diagnostic du « déficit androgénique lié à l'âge » et 2) la définition de la valeur seuil au-dessous de laquelle on peut considérer qu'il existe un « déficit androgénique lié à l'âge ». Le nombre de sujets présentant un "déficit androgénique lié à l'âge » sera variable selon ces critères.

Les premières études ont été réalisées en utilisant le dosage de testostérone totale. Pour éviter d'inclure des sujets ayant des déficits androgéniques secondaires à des pathologies diverses, seuls ont été inclus des sujets « en bonne santé » pour s'intéresser réellement au déficit androgénique uniquement " lié à l'âge ». La notion de «bonne santé » est toutefois relative. Il s`agissait d'exclure les sujets présentant de manière évidente une pathologie chronique ou a fortiori aiguë et prenant des traitements pouvant induire un hypogonadisme. Les études ont ainsi porté sur les sujets vivants à leur domicile et non en institution. La symptoma- tologie fine de déficit androgénique partiel (Tableau 2) n'a pas été spécifiquement étudiée dans ces premières études. En prenant comme valeur seuil la valeur inférieure de la normale chez les hommes jeunes, on constate que la proportion de sujets présentant un déficit androgénique augmente avec l'âge (Tableau 3).

Tableau 3 : Pourcentage d'hommes ayant une testostéronémie $<11$ nmoln [4]

- $20-40$ ans $<1 \%$

- 40-60 ans $7 \%$

- $60-80$ ans $22 \%$

- $>80$ ans $37 \%$

Un élément prédominant dans le choix du type de dosage est lié à l'influence de la protéine de transport de la testostérone dans le sang, la SHBG (Sex Hormone Binding Globulin). Il est clairement établi que, chez les hommes en bonne santé, la SHBG augmente avec l'âge, et que la SHBG module la fraction de la testostérone circulante qui est disponible pour les cellules cibles. La testostérone totale circulante est la somme de la testostérone liée avec une forte affinité à la SHBG, la testostérone liée avec une faible affinité à l'albumine et la testostérone libre. Seules la testostérone libre et la testostérone liée à l'albumine sont facilement disponibles pour les cellules cibles. On qualifie de " testostérone biodisponible " cette somme testostérone libre + testostérone biodisponible.

Il a ainsi été établi que, du fait de l'élévation de la SHBG avec l'âge, la testostérone libre et la testostérone biodisponible diminuent de manière plus marquée avec l'âge que ne le fait la testostérone totale. Ainsi les mesures de ces fractions libres et biodisponibles de la testostérone apparaissent-elles plus à même de faire le diagnostic du « déficit androgénique lié à l'âge » que la mesure de la testostérone totale puisqu'elles ne sont pas sensibles aux variations de la SHBG.

Ainsi, comme nous l'avons indiqué [7], le nombre de sujet présentant un "déficit androgénique lié à l'âge " est plus important si on utilise le dosage de testostérone biodisponible que si l'on utilise le dosage de testostérone totale. Ainsi selon Tenover [10], parmi les hommes en bonne santé de plus de 55 ans, la proportion de sujets ayant des valeurs inférieures à la limite inférieure de la normale des sujets jeunes est de l'ordre de $20 \%$ si on se base sur le dosage de testostérone totale et atteint $50 \%$ si on se base sur le dosage de la testostérone biodisponible.

En ce qui concerne la valeur seuil permettant de retenir le 
diagnostic de « déficit androgénique lié à l'âge », les différents auteurs se basent tous sur la limite inférieure des valeurs normales chez les hommes jeunes.

Il faut toutefois bien reconnaître que cette limite inférieure de la normale des hommes jeunes n'indique pas forcément la valeur au-dessous de laquelle les fonctions androgénodépendantes sont altérées et au-dessus de laquelle elles sont parfaitement normales. D'une part, il nous manque des marqueurs fiables de l'effet physiologique des androgènes qui pourrait nous permettre de déterminer la valeur de testostérone (biodisponible) au-dessous de laquelle le sujet présente effectivement une carence en androgène. Il est possible que la quantité d'androgènes nécessaire puisse varier selon l'effet physiologique que l'on étudie. La valeur de testostérone assurant le maintien de la libido pouvant être différente de celle assurant le maintien de la masse musculaire. D'autre part, les relations entre la testostérone circulante et les effets physiologiques des androgènes ne sont vraisemblablement pas toutes du même type [2]. La relation sexualité testostérone paraît suivre un modèle à seuil alors que la masse musculaire et la masse grasse semblent être liées à la testostérone par une relation de type linéaire, sur une large étendue de valeurs.

\section{Populations étudiées}

Les études ayant permis la mise en évidence du phénomène de « déficit androgénique partiel du sujet âgé » ont été conduites sur des populations d'hommes sélectionnés pour leur état de « bonne santé », et recrutés spécifiquement pour mettre en évidence le phénomène, indépendamment de toute pathologie qui pourrait par elle-même induire un déficit androgénique.

En pratique clinique, le diagnostic du « déficit androgénique lié à l'âge " se pose chez des patients venant consulter pour des symptômes.

La proportion de sujets présentant un « déficit androgénique lié à l'âge » a de fortes chances d'être différente selon les populations étudiées, on pense en particulier aux :

- populations de patients consultant pour des « troubles sexuels »

- populations d'hommes présentant un (ou des) symptôme(s) d'hypogonadisme.

\section{a) Populations de patients consultant pour des « troubles sexuels »}

Les relations entre âge, hormones et sexualité sont complexes. On constate, dans les études épidémiologiques à la fois une augmentation de la fréquence des troubles sexuels chez l'homme avec l'âge et comme on vient de le voir, une diminution de la testostérone circulante avec l'âge. La question est de savoir si ce sont les mêmes sujets qui présentent les troubles sexuels et la baisse de la testostéronémie.
On constate en fait que « troubles de l'érection » et « hypogonadisme » ne coexistent que dans un nombre relativement limité de cas : dans une métaanalyse, Kim et collaborateurs [6] trouvent que le pourcentage de patients consultant pour dysfonction érectile qui ont une concentration de testostérone totale $<300 \mathrm{ng} / \mathrm{dl}$ varie, selon les séries, de 2,1 à $21 \%$ (moyenne 8,3\%). Le motif de consultation « dysfonction érectile » n'est donc que peu spécifique du déficit androgénique.

La relation entre hormones et sexualité chez les sujets avançant en âge a pu être abordée par régression linéaire entre les dosages hormonaux et quantification des comportements et réactions sexuelles comme dans le travail de Davidson et al. [3] qui montre une corrélation significative mais relativement faible $\left(\mathrm{r}^{2}<10 \%\right)$. Ceci indique que le « déficit androgénique » n'explique certainement qu'une part relativement modeste de l'ensemble des troubles sexuels des sujets âgés. Ceci ne nous étonne pas vraiment compte tenu des multiples déterminants de la sexualité humaine.

D'autre part, il apparaît que la relation entre sexualité et testostérone circulante n'est pas linéaire mais procède d'un modèle à seuil. En dessous d'une certaine valeur, le manque de testostérone induit une altération des comportements et réactions sexuelles. Par contre, au-dessus de cette valeur seuil permissive, les comportements et réactions sexuelles ne semblent plus proportionnels à la testostéronémie.

Des manipulations pharmacologiques de la testostéronémie $[1,2]$ ont montré qu'il fallait induire une baisse de la testostérone à un niveau bas pour induire une altération de la sexualité (apparition de troubles sexuels pour une testostéronémie à $0,5 \mathrm{ng} / \mathrm{ml}$ [1] mais pas pour une testostéronémie à $2,5 \mathrm{ng} / \mathrm{ml}[1,2]$. Le maintien de la testostéronémie à 2,5 $\mathrm{ng} / \mathrm{ml}$ a suffi pour éviter une altération de la sexualité, il est vrai chez des sujets jeunes. Il est en possible que cette valeur seuil puisse être différente selon l'âge les sujets et selon le critère étudié (libido, érections nocturnes,...).

Ainsi, les populations d'hommes consultant pour « troubles de l'érection " ne comportent qu'une proportion relativement modérée de patients présentant un « déficit androgénique lié à l'âge ". La valeur seuil de testostéronémie permettant de considérer que les troubles sexuels sont en relation avec le déficit androgénique reste encore mal définie chez le sujet âgé. L'enjeu de sa détermination semble toutefois important dans l'indication des traitements androgéniques. Il est en effet probable que le traitement androgénique ne sera efficace à long terme que si le sujet est effectivement déficitaire et que le déficit représente le principal facteur étiologique des troubles de la sexualité.

\section{b) Populations d'hommes présentant un (ou des) symptô- me(s) d'hypogonadisme.}

D'autres populations peuvent permettre de dépister des 
sujets présentant un " déficit androgénique lié à l'âge », il s'agit des populations de sujets présentant l'un ou plusieurs des symptômes de déficit androgénique. Le problème est que la symptomatologie clinique du déficit androgénique acquis de l'adulte est assez peu spécifique. Il est ainsi apparu judicieux de développer des critères objectifs, basés sur la symptomatologie, dans l'espoir d'améliorer le rendement de dépistage du " déficit androgénique lié à l'âge ». Un questionnaire appelé «ADAM » [8], comporte 10 questions (Tableau 4) principalement basées sur la symptomatologie sexuelle (2 questions), l'humeur (2 questions) et le dynamisme du sujet (5 questions). Une grille d'analyse des réponses (réponse OUI à au moins l'une des 2 questions sexuelles ou à 3 des autres questions évoque un déficit androgénique lié à l'âge) permet d'obtenir une sensibilité de $88 \%$ et une spécificité de $60 \%$ pour prévoir une testostérone biodisponible inférieure à la normale. Un autre questionnaire [9] prend en compte 8 items basés plus particulièrement sur les caractéristiques physiques et les pathologies associées que présentent les sujets (diabète traité, excès pondéral, âge, céphalées, tabagisme, asthme traité, insomnie...). La sensibilité est de $76 \%$ et la spécificité de $49 \%$ pour prévoir une testostérone totale inférieure à la normale. Ces premiers résultats paraissent relativement décevants et n'apportent pas de consensus formel concernant les signes cliniques devant faire pratiquer un dosage de testostérone (biodisponible). Il faut en outre reconnaître que ces tentatives de validation des systèmes de dépistage basés sur la symptomatologie clinique se heurtent, de nouveau, au problème de la définition de(s) valeur(s) de référence permettant de définir précisément le « déficit androgénique ». Il est en effet nécessaire de disposer d'un « gold standard » permettant d'estimer spécificité et sensibilité.

Des études complémentaires apparaissent nécessaires pour déterminer de manière plus précise la (ou les) valeur(s) seuil(s) de testostérone biodisponible indiquant un déficit androgénique relevant d'un traitement substitutif. L'analyse précise des relations entre symptomatologie et niveaux hormonaux, à l'aide de modèles multifactoriels et dans des populations bien caractérisées, devrait permettre de préciser les valeurs seuils indiquant un déficit androgénique.

De plus, il est probable que les résultats des essais thérapeutiques contrôlés aident à définir les critères diagnostiques du « déficit androgénique lié à l'âge », en se basant sur les patients bénéficiant au mieux du traitement substitutif par la testostérone.

\section{CONCLUSION}

Il existe une diminution progressive de l'imprégnation androgénique des hommes pour la seule raison de l'avancée en l'âge. Cette diminution débute assez tôt dans la vie, à partir de la quarantaine mais n'aboutit à un déficit androgé-
Tableau 4 : Test ADAM [8]

1. Avez-vous une diminution de la libido ? (diminution du désir d'avoir des rapports sexuels)

2. Avez vous un manque d'énergie?

3. Avez vous une diminution de la force musculaire ou d'endurance à l'effort ?

4. Avez vous diminué de taille ?

5. Avez-vous noté une diminution de votre joie de vivre?

6. Ettes-vous triste ou irritable ?

7. Vos érections sont-elles moins fortes ?

8. Avez-vous noter une détérioration récente de vos aptitudes sportives?

9. Est-ce que vous vous endormez après le dîner ?

10. Avez-vous noté une détérioration de vos performances au travail?

nique que pour une proportion des hommes. Cette proportion augmente avec l'âge. Le diagnostic du déficit androgénique lié à l'âge fait appel à une méthode d'appréciation de l'imprégnation androgénique de l'individu et se réfère à un seuil, au-dessous duquel on considère qu'il existe un déficit. Compte tenu de l'augmentation de la SHBG avec l'âge, le dosage de testostérone totale n'est pas le meilleur moyen de faire le diagnostic de déficit androgénique lié à l'âge. En utilisant le dosage de testostérone biodisponible ou les formules cherchant à l'estimer par le calcul (index de testostérone libre), la fréquence du déficit androgénique lié à l'âge est plus élevée qu'avec le dosage de testostérone totale. Bien que des études soient en cours pour préciser les relations entre la concentration circulante de testostérone (biodisponible) et les signes et symptômes du "déficit androgénique lié à l'âge ", à l'heure actuelle, les valeurs seuil utilisées pour définir le déficit androgénique lié à l'âge et pour poser l'indication d'un traitement androgénique substitutif, correspondent à la limite inférieure de la normale d'une population d'hommes jeunes.

\section{RÉFÉRENCES}

1. BAGATELL C.J., HEIMAN J.R., RIVIER J.E., BREMNER W.J. : Effects of endogenous testosterone and estradiol on sexual behavior in normal young men. J. Clin. Endocrinol. Metab., 1994, $78: 711-716$.

2. BHASIN S., WOODHOUSE L., CASABURI R., et al. : Testosterone dose-response relationships in healthy young men. Am. J. Physiol. Endocrinol. Metab., 2001, 281 : E1172-E1181.

3. DAVIDSON J.M., CHEN J.J., CRAPO L., GRAY G.D., GREENLEAF W.J., CATANIA J.A. : Hormonal changes and sexual function in aging men. J. Clin. Endocrinol. Metab. 1983, $57: 71-77$.

4. DESLYPERE J.P., VERMEULEN A. : Leydig cell function in 
normal men: effect of age, life-style, residence, diet and activity. J. Clin. Endocrinol. Metab., 1984, 59 : 955-962.

5. HARMAN S.M., METTER E.J., TOBIN J.D., PEARSON J., BLACKMAN M.R. : Longitudinal effects of aging on serum total and free testosterone levels in healthy men. Baltimore Longitudinal Study of Aging. J. Clin. Endocrinol. Metab., 2001, $86: 724-31$

6. KIM C.Y., BUVAT J., CARSON C.C., GOOREN L.J., JAROW J., RAJFER J., VERMEULEN A. : Endocrine and metabolic aspect including treatment. In Erectile dysfunction. 1st international consultation on erectile dysfunction, July 1-3, 1999, Paris. Jardin A., Wagner G., Khoury S., Giuliano F., Padma-Nathan H., Rosen R. eds. Health Publication Ltd, Plymbridge Distributors, Plymouth, 2000 : 207-240.

7. LEJEUNE H. Déficit androgénique lié à l'âge. Andrologie, 2001, 11: 231-239.

8. MORLEY J.E., CHARLTON E., PATRICK P., KAISER F.E., CADEAU P., MCCREADY D., PERRY H.M. : Validation of a screening questionnaire for androgen deficiency in aging males. Metabolism, 2000, 49 : 1239-1242.

9. SMITH K.W., FELDMAN H.A., MCKINLAY J.B. : Construction and field validation of a self-administered screener for testosterone deficiency (hypogonadism) in ageing men. Clin. Endocrinol. (Oxf), 2000, 53 : 703-711.

10. TENOVER J.L. : Testosterone and the aging male. J. Androl., 1997, $18: 103-106$.

\author{
ABSTRACT \\ Androgenic deficiency in aging male: \\ terminology, epidemiology. \\ Hervé LEJEUNE
}

Age-related alteration of reproductive functions differs in men and women. The term "andropause", widely used by the general public, is often replaced by more descriptive terms by the medical and scientific community. Androgen production progressively declines after the age of forty and leads to "androgen deficiency in the aging male" (ADAM) in a proportion of men in good health. This proportion increases with age. As the symptoms are not very specific, confirmation by laboratory tests is necessary. The cut-off used to define ADAM at the present time is the lower limit of the normal values observed in young men. Due to the age-related increase in SHBG, ADAM is more precisely diagnosed by assays taking into account variations of SHBG, such as bioavailable testosterone assay. The percentage of men over the age of 55 with ADAM increases from about $20 \%$ by total testosterone assay to about $50 \%$ with bioavailable testosterone assay.
Key Words: Testosterone, aging, andropause, sexuality, hypogonadism. 